\title{
Pensamiento computacional. Alfabetización digital sin computadoras
}

\author{
Computational Thinking. \\ Digital Literacy without Computers \\ Pensamento computacional. \\ Alfabetização digital sem computadores
}

\author{
Dr. Umberto Roncoroni Osio \\ Profesor principal \\ Facultad de Comunicación \\ (Universidad de Lima) \\ https://orcid.org/0000-0002-9120-2390 \\ Perú \\ Magister Jaime Bailón Maxi \\ Profesor Asociado \\ Facultad de Comunicación \\ (Universidad de Lima) \\ https://orcid.org/0000-0003-3544-3664 \\ Perú
}

Fecha de recepción: 1 de abril de 2020

Fecha de revisión: 3 de abril de 2020

Fecha de aceptación: 31 de mayo de 2020

Fecha de publicación: 1 de julio de 2020

Para citar este artículo: Roncoroni Osio, U., Lavín, E. y Bailón Maxi, J. (2020). Pensa-

miento computacional. Alfabetización digital sin computadoras, Icono 14, 18 (2), 379-405. doi: $10.7195 /$ ri14.v18i2.1570 


\section{Resumen}

El pensamiento computacional es un conjunto de conocimientos en las áreas STEM introducido en los programas educativos para preparar a los estudiantes en la comprensión y el uso de los medios y las herramientas digitales. Sin embargo, no se ha tomado en cuenta que los medios digitales influyen no solo en el desarrollo del conocimiento, sino también en la economía, la cultura, la comunicación y las relaciones sociales. El objetivo es revisar y superar algunas limitaciones del paradigma del pensamiento computacional para aprovechar todo su potencial. Queremos demostrar, mediante la literatura científica y el trabajo de campo, que el pensamiento computacional rescate las humanidades, las manualidades y las tradiciones culturales locales. Para esto se estudiarán: a) la crítica a los fundamentos neopositivistas del tecnocentrismo; b) la naturaleza del medio digital, con énfasis en el software y la creatividad digital, y c) las metodologías y prácticas educativas, entre las que se proponen la máquina de Turing y las shape grammars. Se concluye que, para fortalecer el pensamiento computacional, las maquinarias informáticas no son imprescindibles; más aún, podrían ser contraproducentes para el aprendizaje y la creatividad.

Palabras clave: Computación; Creatividad, educación; Identidad cultural; Software; Tecnocentrismo

\section{Abstract}

Computational thinking has been introduced in schools to provide skills to survive in the digital world, but without proper attention to the fact that digital media are not just means of economic development, but a new way of thinking that modifies culture, communication and social relationships. We will try to demonstrate, with the help of literature, software development and the results of experimental workshops, first, that computational thinking must include humanities and, secondly, analogic skills and cultural traditions. Thus, this article's goal is to rethink the computational thinking framework and overcome its limitations considering the cultural context and especially the rescue of cultural identity. To do this we will follow three main lines of thought: a) the discussion of the limits of technocentrism; b) a proper analysis of the characteristics of software; $c$ ) the analysis of alternative educational solutions like Turing machines and shape grammars. In the conclusions we will show 
that laptops, tablets and smartphones are not indispensable and can even jeopardize learning and creativity.

Key Words: Computation; Creativity; Cultural identity; Education; Software; Technocentrism

\section{Resumo}

O Pensamento Computacional é um conjunto de conhecimentos na área STEM, introduzido em programas educacionais para preparar os alunos para a compreensão e o uso de ferramentas e mídias digitais. No entanto, em geral, não se considera que a mídia digital influencia não apenas o desenvolvimento do conhecimento, mas também a economia, a cultura, a comunicação e as relações sociais. 0 objetivo deste artigo é revisar e corrigir algumas limitações do paradigma do Pensamento Computacional. Queremos demonstrar, com a ajuda da literatura científica e do trabalho de campo, que o Pensamento Computacional deve incluir as humanidades e resgatar práticas educacionais analógicas e tradições culturais locais. Para isso, serão estudados: a) críticas aos fundamentos neopositivistas do tecnocentrismo; b) a natureza do meio digital, com destaque para software e criatividade digital; c) metodologias e práticas educacionais nas quais as Gramáticas de Touring Machine e Shape são propostas. Ao final, se conclui que, para fortalecer o pensamento computacional, o maquinário para computadores não é essencial e pode até ser contraproducente para $o$ aprendizado e a criatividade.

Palavras chave: Computação; Criatividade; Educação; Tradições culturais; Software; Tecnocentrismo

\section{Introducción}

La economía de redes, la fabricación digital, la robótica, la inteligencia artificial y las redes sociales generan cambios disruptivos en las empresas, en la producción, en el marketing y en la comunicación. En el campo educativo, las instituciones, los docentes y los estudiantes encuentran dificultades para relacionarse con la revolución digital y responder eficazmente a las complejas transformaciones políticas, económicas y sociales. Tanto es así que, luego de muchos entusiasmos iniciales, se 
comienza a reconocer que la informática educativa no está dando los resultados esperados (Buckingham, 2006; Dussel y Quevedo, 2010; Denning, 2017; Wolf, 2018). Hay dos razones principales que explican este desencuentro.

En primer lugar, las tecnologías, y en especial las digitales, no son política y culturalmente neutrales. Como señalaron Deleuze (2006) y Flusser (1984), hoy las máquinas son sistemas complejos que capturan al usuario en una cadena de procesos ocultos y cada vez más dependientes del poder económico y político. Por tanto, los medios digitales deben ser leídos considerando las contradicciones del sistema capitalista contemporáneo: las dinámicas de la innovación democrática y la realidad de los monopolios. La primera se expresa en la utopía de internet de Barners Lee y en la cultura hacker y del software libre (Stallmann, 2002; Lanier, 2014); la otra consiste en los nuevos sistemas de control y censura de los monopolios digitales como Google o Facebook.

En segundo lugar, aún no existe una idea suficientemente clara de los procesos, las propiedades y los efectos socioculturales de los medios digitales. Se acelera el desarrollo tecnológico como consumo, pues las TIC son consideradas herramientas prácticas y operativas sin connotaciones culturales, pero se ignora la naturaleza creativa del medio digital y el hecho de que las aplicaciones comerciales condicionan a los usuarios, debido a la mediación de las interfaces, en un universo simbólico y en un paradigma epistemológico y operativo único y globalizado.

Las recientes reformas curriculares en Europa o Estados Unidos (Ministero dell' Istruzione, dell'Universitá e della Ricerca [MIUR], 2010; Johnson, Adams Becker, Estrada y Freeman, 2015) han intentado mejorar el uso de las TIC introduciendo en los currículos de primaria y secundaria el pensamiento computacional (Wing, 2006; Aho, 2012). Pero esta solución padece de las mismas limitaciones que pretende superar. En efecto, los medios digitales establecen un verdadero zeitgeist que, trascendiendo el dominio del aparato tecnológico, requiere principios neurolingüísticos, cognitivos, computacionales y, sobre todo, filosóficos y estéticos. Estos contenidos trascienden el ámbito de las disciplinas STEM y apelan a las humanidades y a los saberes y habilidades analógicos, precisamente aquellos que las TIC pretenden reemplazar. En este artículo discutiremos algunas cuestiones cru- 
ciales desde el punto de vista educativo: los límites del "tecnocentrismo" (Papert, 1980) y del "solucionismo tecnológico" (Morozov, 2014) $i^{1}$, la naturaleza creativa de los medios digitales; y la importancia de la identidad cultural y de las prácticas educativas tradicionales, para lo cual más adelante proponemos dos soluciones tecnológicas alternativas.

\section{Método}

\subsection{Problema de la investigación}

En su formulación original, Wing (2006) plantea que el pensamiento computacional tiene por finalidad preparar a los futuros profesionales (no solo a los de la ingeniería informática) para la sociedad red y el mundo digital. En este sentido, el pensamiento computacional se centra en los conocimientos y las competencias STEM (acrónimo en inglés para referirse a las habilidades en las ciencias, tecnología, ingenierías y matemáticas).

Sin embargo, algunos investigadores como Denning (2017), han señalado que, con excepción de los especialistas en informática, el pensamiento computacional resulta de poca utilidad práctica y educativa. Sobre el mismo concepto, Guzdial, Kay, Norris y Soloway (2019) han indicado que sus contenidos son genéricos e incompletos para una efectiva contribución a la innovación de las políticas educativas, sobre todo, en lo que se refiere a la creatividad, la inclusión y la sostenibilidad (Fonseca, 2005; Varma, 2006). Algunos estudiosos incluso han observado que la teoría del pensamiento computacional carece de una clara visión de las peculiares propiedades de los medios digitales esenciales para una real innovación educativa (Buckingham, 2006; Manovich, 2013).

\subsection{Objetivos, hipótesis y justificación}

Nuestro objetivo es revisar y superar algunas limitaciones del paradigma del pensamiento computacional para aprovechar todo su potencial, bajo el supuesto que es indispensable la inclusión de las humanidades, el rescate de las prácticas educativas analógicas y las tradiciones culturales locales, a partir del desarrollo de tres tareas. 
La primera es la revisión del concepto de pensamiento computacional, centrado en el desarrollo de capacidades cognitivas STEM, originalmente elaborado por Wing (2006). El pensamiento computacional no debería reducirse a las ciencias de la computación, pues no todo es computable; incluso aquello que lo es depende del devenir del contexto sociocultural. Por esto necesitamos una visión más compleja de los procesos mentales de emisión de juicios y de resolución de problemas implicados en la computación. Aquí el pensamiento computacional se construiría en el límite de lo analógico y lo digital. El reto es que estos dominios, sin entrar en conflicto, colaboren enriqueciéndose mutuamente.

La segunda tarea es desvelar las características más importantes de los medios digitales dentro de su conexión con las ciencias de la complejidad, el lenguaje y los saberes humanísticos. Sin la inclusión de aspectos más humanos en la enseñanza, como han señalado Reiman y Maday (2017), el pensamiento computacional no puede superar lo meramente operativo y contingente.

Finalmente, la tercera es proponer una alternativa de alfabetización digital sin computadoras, para lo cual presentaremos las posibilidades y ventajas educativas de la máquina de Turing y de las shape grammars $^{2}$.

La forma de aprender los medios digitales que proponemos es especialmente relevante para un país como el Perú, porque permite incorporar los valores culturales y los saberes ancestrales de sus diversas regiones. Esto ayudaría a cerrar brechas y mejorar la inclusión de los estudiantes de zonas alejadas con escasos recursos económicos.

\subsection{Metodologías, materiales y resultados}

El contenido de este artículo deriva de las investigaciones desarrolladas en el Instituto de Investigación Científica de la Universidad de Lima (IDIC) en los últimos tres años. Desde el punto de vista teórico, se hizo una revisión crítica de la literatura indexada y se trabajó en el desarrollo de contenidos para los cursos y talleres y en el diseño de software educativo experimental ${ }^{3}$. Concretamente, se han desarrollado dos aplicaciones en C\#: un simulador de la máquina de Turing y un 
simulador de la yupana, la calculadora inca4. Para el diseño de la interfaz y de las funciones, se han capitalizado las actividades de los talleres.

El trabajo de campo ha sido esencial para poner a prueba nuestras hipótesis metodológicas y didácticas y ha revelado un componente fundamental para mejorar la eficacia educativa de la tecnología: el desarrollo manual previo mejora el entendimiento de las simulaciones digitales y su evaluación crítica. Esto vale tanto para los estudiantes como para los investigadores y programadores que diseñan aplicaciones educativas.

\subsection{Estructura del artículo}

El primer tópico que discutiremos será la crítica al tecnocentrismo, considerando el contexto social y, especialmente, ciertos tópicos de las ciencias de la complejidad que contribuyen al desarrollo de las ciencias de la computación. Luego, haremos un análisis de las características peculiares de los medios digitales: el lenguaje numérico y su dimensión de metamedio (Kay, 1984).

En el siguiente apartado se tomarán en cuenta estas reflexiones para otorgar relevancia educativa al paradigma del pensamiento computacional en sentido inclusivo, creativo y sostenible.

En el último apartado, presentaremos las ventajas de las máquinas de Turing y de las shape grammars. Las conclusiones se formulan alrededor de una paradoja: para desarrollar un verdadero pensamiento computacional necesitamos las humanidades, los contenidos y las didácticas que el tecnocentrismo casi ha logrado suplantar. Más aún, las maquinarias informáticas son prescindibles y hasta contraproducentes para el aprendizaje y la creatividad.

\section{Discusión}

Las TIC se consideran herramientas dedicadas principalmente al desarrollo científico y económico y a la gestión de las instituciones educativas, lo que explica el enfoque STEM del pensamiento computacional. El tecnocentrismo tiene alguna ra- 
INNOVACIÓN TEÓRICA

zón de ser; sin embargo, los medios digitales no son solamente herramientas utilitarias, sino también agentes importantes de las contradicciones de la sociedad red.

\subsection{Los límites del tecnocentrismo}

Detrás de ciertas inconsistencias de las reformas y de los escasos éxitos educativos de los medios digitales, está el desencuentro entre la tecnociencia, la sociedad del conocimiento y los problemas de sostenibilidad del modelo de desarrollo neoliberal. Estos son los ejes principales que seguiremos para entender el rol del pensamiento computacional en las dinámicas que ya hemos delineado.

\subsubsection{El auge de la complejidad y el pensamiento computacional}

El marco teórico del pensamiento computacional se basa en una idea de ciencia que no refleja la puesta en discusión y los cuestionamientos del realismo y del determinismo neopositivista, pues hoy la ciencia reconoce, por ejemplo, el caos, el tiempo y los sistemas dinámicos. El pensamiento computacional debería, entonces, incluir los algoritmos generativos, los fractales y la vida artificial (figuras 3, 4 y 6). Tanto es así que estos principios de la complejidad sustentan el desarrollo de nuevas herramientas cognitivas que tienen aplicaciones concretas en la computación, en la industria informática y en la producción audiovisual, como los videojuegos.

Por otro lado, en la filosofía se avala la crítica al método científico y se da pase a la hermenéutica, al posestructuralismo y a la lingüística. Particularmente significativos son los aportes de las neurociencias y de la psicología cognitiva acerca de la relación entre caso, emociones y racionalidad (Damasio, 2005; Kahneman, 2012). Aquí se pone en tela de juicio la ilusión del mundo lógico y ordenado en el que confía la informática. Esta suerte de deconstrucción del método cartesiano es clave para entender las limitaciones del big data y del machine learning (Rahimi, 2019; Zador, 2019). La cientificidad de estas tecnologías es una ilusión, porque, por ejemplo, la selección de los datos y los parámetros del aprendizaje de las redes neuronales no tienen mayor sustento que las creencias de los programadores: "somos propensos a sobrestimar lo que entendemos del mundo y a subestimar el papel del azar en los acontecimientos" (Kahneman, 2012, p. 27). 
Si tomamos en cuenta estos factores, es justificado afirmar que el pensamiento computacional, en cuanto instrumento conceptual, debería ayudar a los futuros ciudadanos a reconocer las polaridades que alimentan las contradicciones de la sociedad red: la lógica rígida y mecanicista de la computación y la impredecible complejidad de los acontecimientos socioculturales.

\subsubsection{Los desencuentros de la sociedad del conocimiento}

Las contradicciones entre el tecnocentrismo, el pensamiento computacional y la complejidad sociocultural se reflejan también en la sociedad del conocimiento y en las falacias del "solucionismo" tecnológico (Morozov, 2014). La primera ilusión que se desvanece es la del libre acceso al conocimiento en internet, que admitía preguntas como ¿Para qué investigar o reflexionar? y ¿Para qué construir teorías? Para el solucionismo tecnológico la teoría es inútil, porque confía en que los grandes servidores pueden extraer conocimiento verdadero y construir tendencias que se pueden verificar con exactitud simplemente procesando la enorme cantidad de datos disponibles (Han, 2014).

Kurz (2003), Deleuze (2006), Rifkin (2013) y Lanier (2014) han mostrado cómo las relaciones entre los medios digitales, la política y la economía generan nuevas formas de poder y control. Los monopolios de internet - Google o Facebook- ponen ulteriormente a prueba el dato como correspondencia objetiva a los fenómenos de la realidad. El proceso se consuma a través de la invasión de nuestra privacidad y el control de los criterios de acceso a los datos, que en realidad recibimos filtrados por las prioridades de dichos monopolios.

En este sentido, el perfil tecnocéntrico del pensamiento computacional es inadecuado y autorreferencial, en tanto que pretende solucionar computacionalmente problemas cuyos orígenes son las mismas estructuras de la computación.

\subsubsection{Desarrollo, políticas educativas y sostenibilidad}

El último paso para reconsiderar los contenidos del pensamiento computacional es averiguar su compatibilidad con las demandas del desarrollo y del mercado. 
INNOVACIÓN TEÓRICA

Respecto al desarrollo tecnológico, se ha señalado la falta de inclusión y sostenibilidad y la manifestación de nuevas formas de colonización que caracterizan el neoliberalismo globalizado (Harris et al., 2018; Varma, 2006). La lógica empresarial atrapa a los estudiantes en lo contingente y los obliga a aprender habilidades o saberes que serán rápidamente expropiados por la inteligencia artificial y los robots.

En cuanto a las políticas educativas, la instrucción formal entra en competición con los "aprendizajes invisibles" (Cobo Romaní y Moravec, 2011), pues las TIC se aplican sin considerar que los jóvenes aprenden contenidos digitales fuera de las instituciones educativas tradicionales: celulares, consolas e internet. Además, la calidad del software educativo está lejos del nivel del mercado, lo que justifica el rechazo de los estudiantes, acostumbrados a los juegos AAA para Xbox o PlayStation (Buckingham, 2006). Los aprendizajes invisibles, la aceleración científica y tecnológica, y las ofertas educativas virtuales requieren del sujeto la disposición al autoaprendizaje, la autodisciplina educativa y la autonomía en la navegación del saber en lo que por ahora no tienen ni mentores ni tutores. Nadie explica a los jóvenes cómo buscar información en internet ni los criterios éticos, sociales y políticos para averiguar su calidad; es decir, el pensamiento crítico y socialmente contextualizado. Esto se adquiere solo mediante el conocimiento histórico, filosófico y literario. Para ofrecer al estudiante la posibilidad de adquirir estas competencias esenciales, el pensamiento computacional debe reencontrar de alguna forma las ciencias humanas.

\subsubsection{Complementos para una epistemología del pensamiento computacional}

¿Qué indicaciones podemos deducir para corregir el marco teórico del pensamiento computacional? En primer lugar, hay que tomar en cuenta la manifiesta relación entre las ciencias de la complejidad, la computación y la creatividad. La industria de la producción audiovisual digital, por ejemplo, requiere de sus profesionales habilidades fundadas en la creativa hibridación de ciencia, letras y arte. En segundo lugar, las idiosincrasias de la sociedad del conocimiento, los peligros de los monopolios y la inteligencia artificial, desbordando los parámetros cuantitativos, apelan a las humanidades, pues requieren verificar fundamentos, criterios y fines del quehacer tecnológico. Considerando todo esto, es claro el peligro del pragmatismo mal entendido y de la miopía computacional que rechaza las humanidades. 
En relación con nuestra hipótesis, podemos concluir que los conocimientos y las habilidades necesarias para comprender los medios digitales exceden lo que las ciencias de la computación y la maquinaria informática pueden ofrecer. En el siguiente apartado, analizando las peculiares propiedades de los medios digitales, mostraremos con mayor evidencia los límites del tecnocentrismo y el potencial de la complejidad.

\subsection{Acerca de la naturaleza de los medios digitales}

El pensamiento computacional se propone como un conjunto de saberes y competencias para sobrevivir en escenarios diseñados por los avances tecnológicos; sin embargo, su efectividad es limitada en cuanto se basa en una visión superficial y hasta incorrecta de los medios digitales y de sus requisitos epistemológicos y pedagógicos. El objetivo de este apartado es individuar tres aspectos de los medios digitales que el marco del pensamiento computacional debería necesariamente considerar: el proceso computacional en sí, la materia digital y las capas mediáticas del software en cuanto metamedio.

\subsubsection{El proceso computacional}

Una de las razones por la cual las computadoras son mal utilizadas en la práctica educativa (Buckingham, 2006; Wolf, 2018) es que ni sus propiedades ni las características del proceso computacional son comprendidas a cabalidad, tal como Turing ha mostrado con la máquina de Turing. En primer lugar, la computación no es cálculo, sino un proceso iterativo que actúa mediante el posicionamiento, la lectura y la escritura de ceros y unos en un espacio lineal. En segundo lugar, el cómputo no tiene ni inteligencia ni conciencia, pues es indiferente al contenido informativo que lee o escribe. La magia del proceso computacional consiste precisamente en lograr resultados correctos a pesar de no saber ni calcular ni leer. Por esto, para diseñar un proceso computacional, además de los contenidos STEM, se necesitan fantasía, diseño, razonamiento lingüístico y manipulación simbólica, contenidos que no están del todo incluidos ni siquiera en su extensión STEAM (Henriksen, 2017). 
INNOVACIÓN TEÓRICA

\subsubsection{La materia digital}

Para comenzar, hay que hablar de las mitologías que ofuscan la relación entre lo analógico y lo digital. Los medios digitales no son, en cuanto digitales, una novedad, porque son siempre simulaciones de los procesos analógicos. Por lo tanto, no se comprenden sin conocer a sus ancestros o los modelos en el mundo físico, en la historia y en la naturaleza. Dos evidencias ejemplares son el diseño 3D, que se basa en la perspectiva renacentista y en la elaboración de las imágenes en mapa de bits, cuyos algoritmos simulan las técnicas artísticas y fotográficas tradicionales, como los efectos de la aeropittura futurista utilizados para los filtros de programas como Photoshop (figura 1).
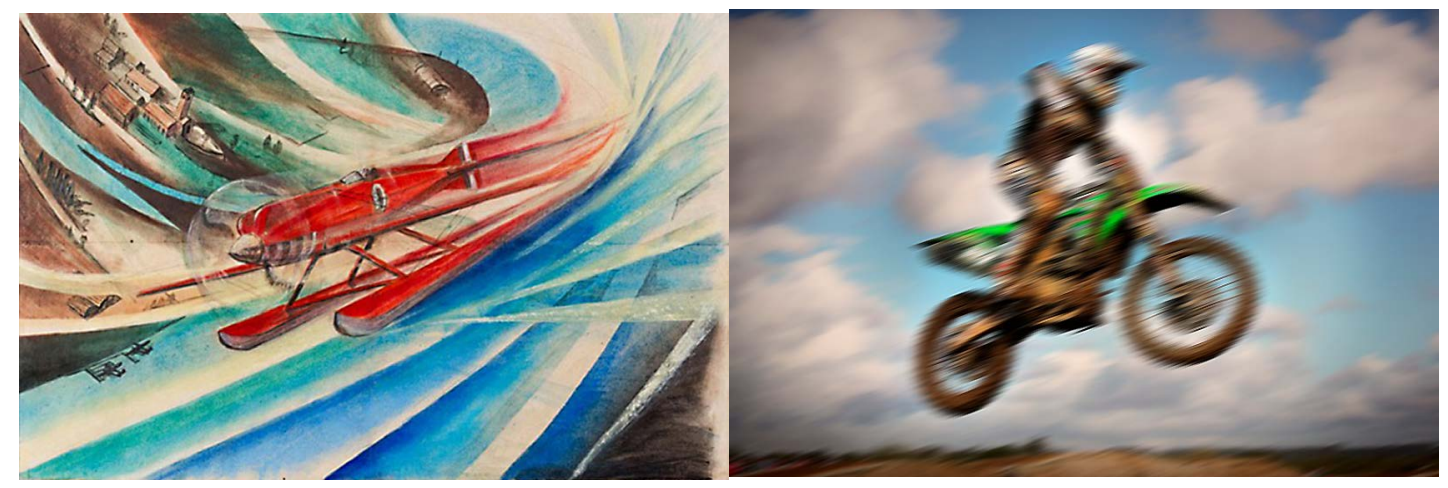

Figura 1: A la izquierda: Sansoni, G. (1930). Aeropittura. Los futuristas estudiaban este efecto dentro un avión en picada, ya que, por la fuerza centrífuga, la visión se hace borrosa. Fuente: Italian Ways. A la derecha, filtro desenfoque de movimiento. Fuente: elaboración propia.

La otra posibilidad característica del medio digital que no se aprovecha es la unificación de todas las impresiones que comunican los sentidos (formas, colores, sonidos) en una información numérica binaria. Esta es imperceptible, pues las salidas audiovisuales a través de monitores o parlantes pertenecen al dominio analógico. La ignorancia de este detalle tan importante hace que se siga "explicando" lo digital mediante sus instancias analógicas, lo que oculta el contenido original y propiamente numérico dentro una caja negra. 
Por esto resulta muy difícil lograr que los estudiantes comprendan a fondo lo digital y aprovechen aquellos procesos que resultan imposibles en el mundo analógico. Así, la retroalimentación entre letras, imágenes, sonidos y cálculos, que redefine la idea analógica de la multimedia en el plano abstracto del algoritmo, del código y de los datos.

Aquí lo digital permite utilizar un proceso como contenido (códigos y algoritmos), medio (textos, imágenes o sonidos) o herramienta (las aplicaciones). Desde el punto de vista de los contenidos educativos, es esencial comprender que lo digital no existe sin el soporte de las técnicas, las recetas y los ingredientes del mundo físico.
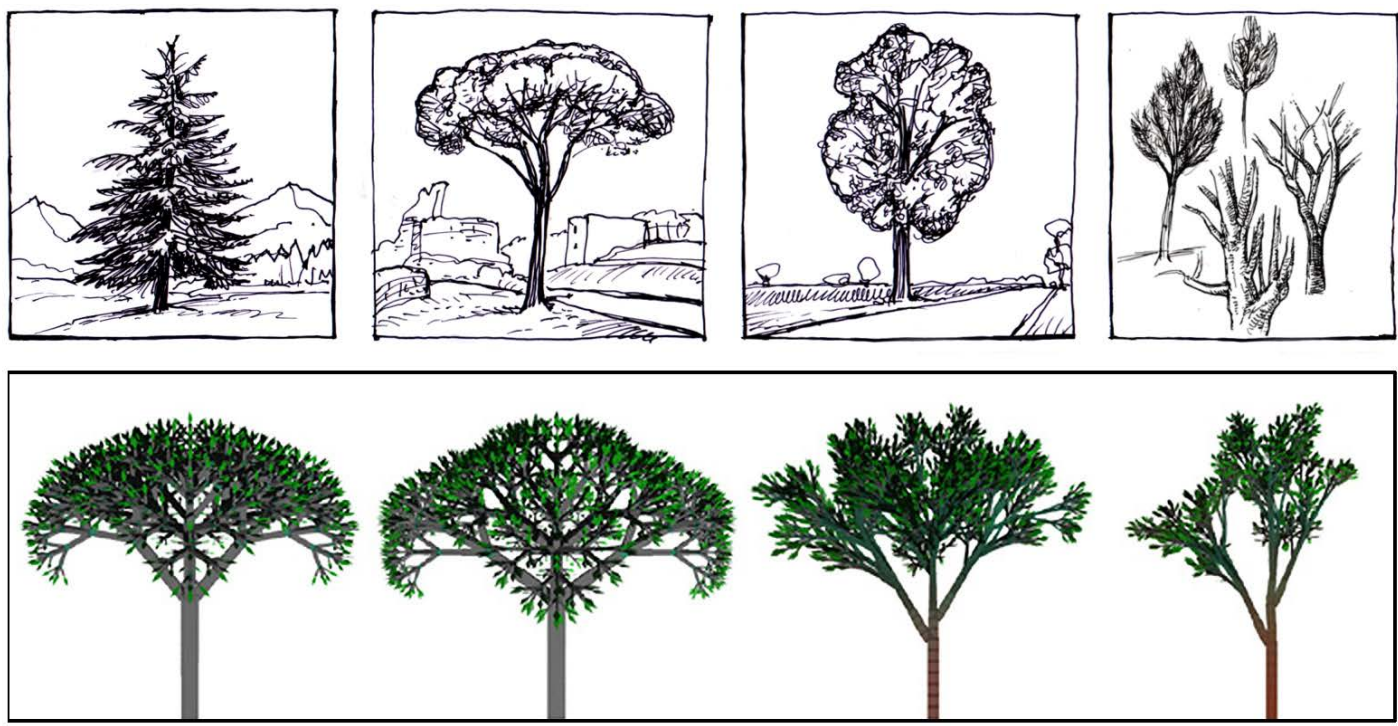

Figura 2: Desde el dibujo analógico hasta los modelos generativos 3D. La modelación 3D se basa en la observación de los procesos y fenómenos naturales que el dibujo del natural hace comprender de forma óptima. En computación se simulan mediante fractales, shape grammars o vida artificial. Fuente: elaboración propia. 
Un ejemplo es la generación de los mundos virtuales 3D para los videojuegos 0 las películas de animación (figura 2), que se basa en los procesos naturales orgánicos e inorgánicos, como los fractales y los procesos generativos ${ }^{5}$ estudiados en las ciencias de la complejidad (Flake, 2000).

\subsubsection{Las capas del software}

De acuerdo con lo anterior, una conclusión obvia es que el medio digital propiamente dicho es el software con sus múltiples capas: es un almacén y canal que transporta datos e informaciones, una herramienta operativa, un patrimonio de conceptos y saberes, y es un medio de comunicación, pues el código y las interfaces son textos. Y el código, en cuanto texto, permite dirigir los procesos que se ejecutan en la computadora, siguiendo normas lingüísticas que posibiliten su escritura y lectura (Fishwick, 2008; Manovich, 2013).

Desde aquí se deduce la característica única del software, como subrayó uno de los padres de la computación, Alan Kay (1984, p. 52-59): “It is not a tool, although it can act like many tools. It is the first metamedium, and as such it has degrees of freedom for representation and expression never before encountered and as yet barely investigated" ${ }^{6}$. Es decir, el software es una herramienta que puede crear otras herramientas.

La creatividad es su rasgo esencial. La prueba son los lenguajes de programación, que pueden crear otro lenguaje de programación (Python está escrito en el lenguaje de programación $\mathrm{C}$ ), con el cual se puede crear una aplicación como un videojuego o una DAW (Digital Audio Workstation o estación de trabajo de audio digital). Pero esta característica sigue subestimada si no del todo ignorada, y, por lo tanto, desaprovechada para la educación.

\subsection{El pensamiento computacional 2.0}

En síntesis, el pensamiento computacional debe brindar a los futuros profesionales herramientas críticas y competencias específicas para explotar creativamente las propiedades del software como proceso primario de todas las aplicaciones digitales. En primer lugar, deben ser habilidades para el autoaprendizaje permanente 
que las transformaciones sociales, científicas y tecnológicas imponen a las nuevas generaciones y que, en la mayoría de los casos, se hace sin las instituciones educativas tradicionales. Además, el pensamiento computacional debe incluir las características y los saberes propios de los medios digitales, pues estos son el motor de la evolución del paisaje cultural tecnológico.

En efecto, para las aplicaciones educativas del pensamiento computacional existen, como la literatura ha evidenciado (Fonseca, 2005; Varma, 2006; Denning, 2017; Guzdial et al., 2019), dos riesgos: o bien difuminar sus contenidos dentro de una genérica interdisciplinariedad que le quita el derecho a proponerse como una nueva disciplina, o bien cerrarse en contenidos especializados que restringen el universo de su público objetivo.

En segundo término, para el pensamiento computacional se presenta un escenario compuesto por combinaciones de contenidos interdisciplinarios apoyados en lo analógico, y por los conocimientos específicos del universo informático. El reto de manejar los medios digitales se revela en toda su complejidad, pues necesitamos coordinar dominios epistemológicos que van desde las ingenierías hasta las artes, lo que requiere del individuo un esfuerzo cognitivo enorme y de las instituciones educativas cambios más complicados y estructurales. Es aquí cuando el tecnocentrismo - que consiste precisamente en pensar los medios digitales de modo instrumental, sin creatividad y sin relación con los contextos socioculturales-impide el avance del pensamiento computacional.

\subsubsection{Pensamiento computacional 2.0 y creatividad}

Desde el punto de vista del pensamiento computacional en la práctica educativa, lo analizado muestra que se requiere priorizar las competencias críticas, desmitificando el aura del aparato tecnológico. Asimismo, se pone en claro que el pensamiento computacional tiene dos grandes retos: diseñar las nuevas competencias para manejar la convergencia de medios y lenguajes, y explotar la peculiar dimensión creativa del software. En ambos casos, hay que saber jugar con los referentes analógicos y con las dificultades completamente nuevas del código. Por lo tanto, no debemos enseñar el medio digital mediante las aplicaciones comerciales dedicadas a una tarea específica, como 
Excel o Photoshop, porque encierran al usuario en el marco establecido por el programador y porque hacen cosas que conceptualmente se pueden realizar de manera analógica. La única diferencia sería cuantitativa: menos tiempo y menores costos.

Con esta limitación, se pierde el potencial creativo propio del medio digital. Instituir el coding como competencia es un buen avance, pero es insuficiente si no se completa tomando en cuenta las exigencias del software.

\subsubsection{Information literacy y computational creativity}

La complejidad del pensamiento computacional corresponde a la naturaleza compleja del software: medio creativo o expresivo, y herramienta de investigación, producción y comunicación. En la primera modalidad, el dominio de lo digital es general, transdisciplinario y fundamentalmente humanístico; en la segunda, se ubica en las ciencias de la computación y constituye un conjunto de disciplinas cercanas a las matemáticas e ingenierías. Ahora bien, es evidente que el pensamiento computacional no puede resolver las dos culturas de la misma manera.

Para individuar contenidos y métodos adecuados a la dimensión sociocultural del pensamiento computacional, regresamos al concepto de information literacy propuesto por Shapiro y Hughes (1996). Desde este punto de vista, se considerarían aquellos conceptos y métodos de los medios digitales que se podrían generalizar e incluir en cualquier asignatura. En otras palabras, la information literacy consistiría en lo siguiente: a) conectar los contenidos de cada disciplina con sus apropiadas soluciones tecnológicas de acuerdo con las propiedades del medio digital, y b) aprender a utilizar la computación como auxilio creativo y expresivo. Precisamente, esto es lo que propone la computational creativity, que, en efecto, algunos consideran una nueva rama del pensamiento computacional (Mazzone y Elgammal, 2019).

Considerando las características de la cultura digital y del software como metamedio creativo, resulta que los aparatos informáticos y los gadgets digitales en la educación son una mediación innecesaria que interfiere con el potencial 
de la computación, su inclusión y sostenibilidad. Esta afirmación se sustenta tanto desde el punto de vista de la information literacy como desde las ciencias de la computación en las que hemos dividido el campo del pensamiento computacional.

\subsubsection{Cultura digital sin computadoras}
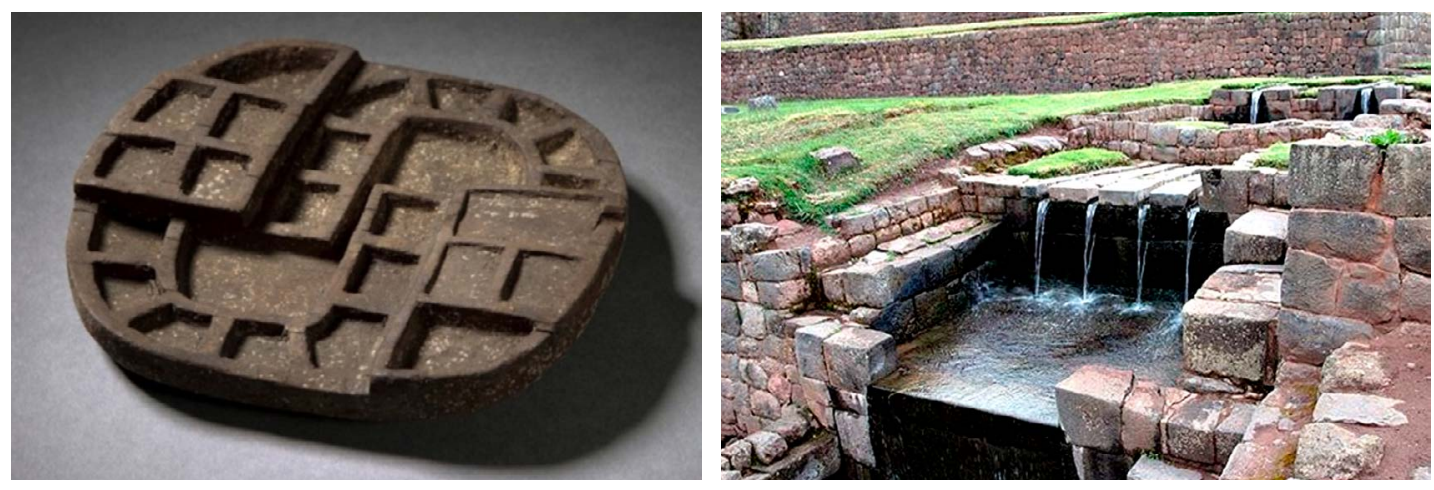

Figura 3: Izquierda: Lorenzo, E. (2019). Yupana. Fuente: Yupana y quipu. Derecha: Gil, A. (s. f.). Tipón, Perú. Fuente: fotoAleph. Las instalaciones de Tipón son un ejemplo de computación natural para la hidráulica. Son efectivamente máquinas de estado finito arquitectónicas relacionadas con en el paisaje.

El uso y abuso de aplicaciones digitales -en Latinoamérica casi siempre importadas - impone un modelo de cultura digital limitado al consumismo pasivo, que incluso permite una expresión tecnológica de la colonización (Fonseca, 2005; Varma, 2006; Lanier, 2014). Con esto se le resta espacio a la autonomía educativa, la creatividad de los estudiantes y los recursos para la investigación de software original.

Poner límites al uso de la maquinaria de las TIC fortalece la identidad cultural, pues dejaría espacio a la etnomatemática y a la etnocomputación, como la yupana (figura 3) o los kenés shipibos peruanos (figura 4) (Apaza Luque, 2017) $)^{4}$. 

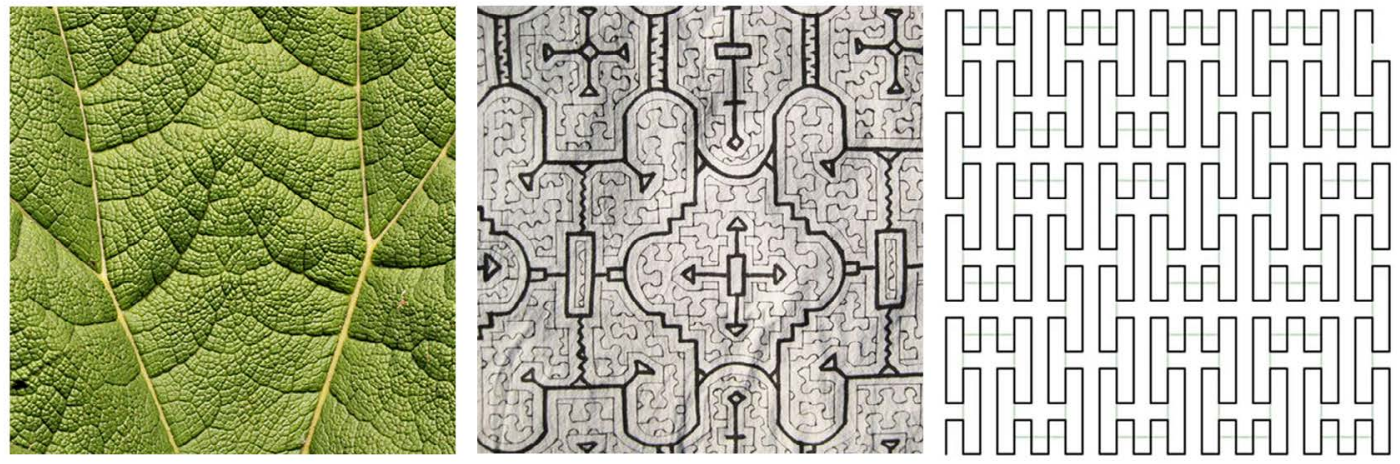

Figura 4: Continuidad entre naturaleza, tradición y computación. Nervaduras de una hoja, dibujo kené y curva de Peano. Fuente: elaboración propia.

Estos artefactos y tecnologías ancestrales permiten que los estudiantes hagan una conexión espontánea entre el medioambiente, las tradiciones culturales y la computación, puesto que se pueden armar y operar manualmente con recursos que son parte de su entorno. Para el diseño del proceso computacional, las mejores herramientas son el arte y el dibujo a mano alzada porque, primero, transmiten sin interferencias la idea al desarrollo del proceso, $\mathrm{y}$, segundo, se pueden aplicar trasversalmente en diferentes asignaturas. Con esto, los contenidos más abstractos se vuelven significativos, refuerzan la autoestima y, por ende, el impulso creativo de los estudiantes.

En lo que se refiere a las ciencias de la computación, el pensamiento computacional, como señalaron Wing (2006) y Aho (2012), comprende el pensamiento algorítmico y recursivo, la lógica, la gestión de datos y el diseño de sistemas interactivos. En este sentido, define correctamente los contenidos STEM que preparan al estudiante para gestionar de modo profesional las aplicaciones informáticas o la programación. Pero, aun así, el modelo STEM resulta insuficiente. Recuperemos lo esencial: la naturaleza de metamedio de la computación y la diferencia entre el diseño de tecnologías originales y el consumo de aplicaciones ya hechas. Incluso en el marco restringido de las ciencias de la computación, aparece la naturaleza esencialmente creativa del medio digital; por lo tanto, el pensamiento computacional no tendría mucho que ver con soluciones TIC prefabricadas. Como es evidente, esto provoca problemas metodológicos en relación con su enseñanza en las clases. No obstante, existen tecnologías, todavía poco aprovechadas, que ofrecen alternativas educativamente viables. 


\subsubsection{Métodos y materiales para el pensamiento computacional 2.0}

En las reformas educativas de los Estados Unidos y de varios países de la Unión Europea, el primer cambio curricular para mejorar las competencias digitales de los estudiantes ha sido introducir el coding. Esta decisión, en la opinión de varios educadores e investigadores (Schmidt-Crawford, 2018; Guzdial et al., 2019), se valida porque programar mejoraría la lógica, el design thinking, la gestión de proyectos y las habilidades blandas tan necesarias para el trabajo colaborativo y grupal. Sin embargo, el coding, para ser efectivo y significativo, demanda muchos conocimientos previos y demasiado tiempo para aprender los lenguajes utilizados (incluso aquellos visuales como Scratch). Sobre todo, necesita algo que no todos los estudiantes tienen: el compromiso y la pasión. Y, finalmente, no hay pruebas de que el coding mejore realmente las habilidades STEM y que sea útil para aquellos que no estudiarán informática (Schmidt-Crawford, 2018). En este sentido el coding, sin negar su utilidad educativa, no puede ser el principal medio de acercamiento al mundo digital.

Una alternativa susceptible de ser investigada son aquellos procesos computacionales que desarrollan el diseño algorítmico y los principios de la programación de forma más simple, suavizando la transición entre los procesos analógicos y la aplicación informática. Lo aprendido mediante el desarrollo de software y los resultados de los talleres experimentales nos permiten sugerir las máquinas de Turing y las shape grammars, como se explica más abajo.

Ambos procedimientos se llevan a cabo mediante una sintaxis minimalista y un lenguaje visual compacto, con mecanismos que se aprenden rápidamente y que se pueden desarrollar a mano alzada, lo que lleva a comprender los procesos de la programación de manera más intuitiva (figuras 5 y 6).

La genial simplicidad de estos procesos permite resolver cualquier tipo de problema computable solo con el auxilio de nuestro cerebro, nuestra mano y materiales simples, como lápiz, papel u objetos de uso cotidiano. La implementación informática, pues, sirve solo para ejecutar el proceso más rápidamente; pero, precisamente por esto, oculta sus procesos cognitivos y creativos dejando al usuario frente a las limitaciones de las interfaces. 

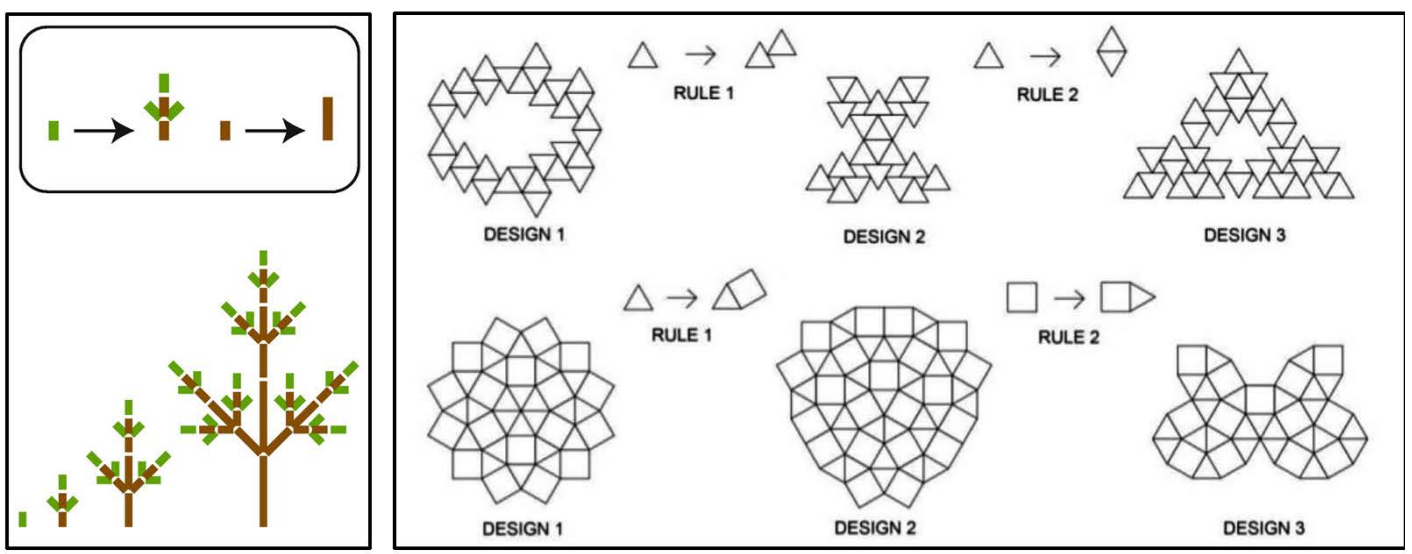

Figura 5: Ejemplos de shape grammars. A la izquierda, Prusinkiewicz, P., Cieslak, M., Ferraro, P. y Hanan, J. (2018). Modeling plant development with L-systems. Fuente: Springer Link. Derecha: Shen, G. (2014). Shape grammar exercises. Fuente: Slide Share.

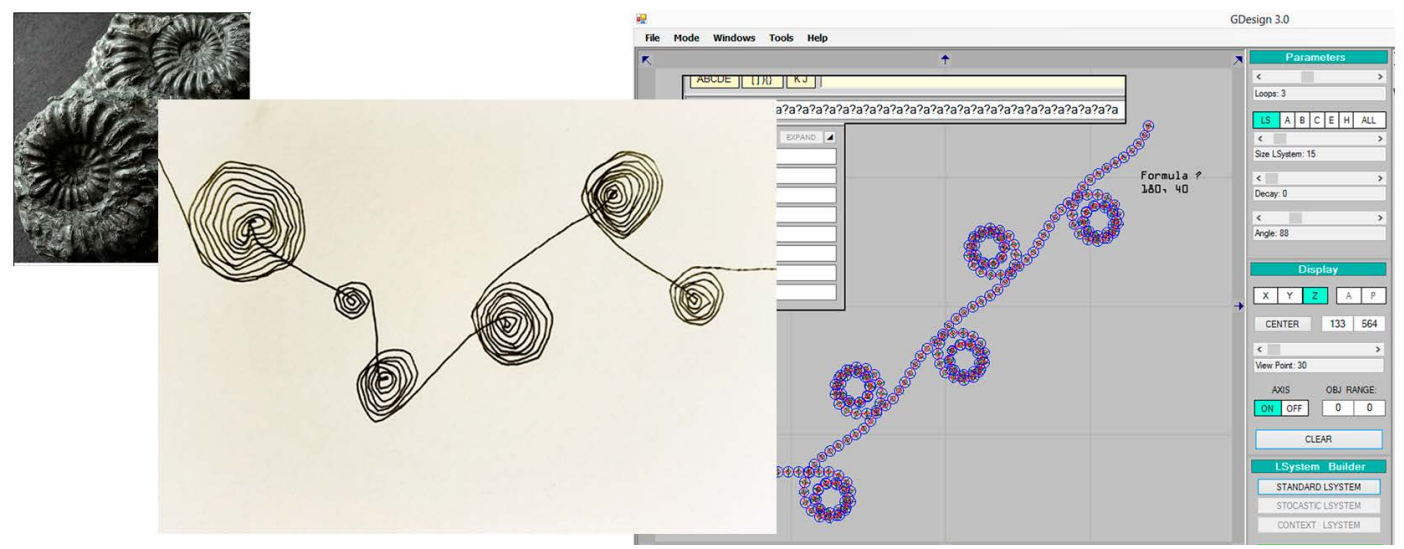

Figura 6: Desde las formas naturales al dibujo procedural y al software generativo.

Fuente: elaboración propia a partir de un dibujo de Crousse,

V. (2018) y software de Roncoroni, U. (2017).

\section{Conclusiones}

Este artículo analiza ciertas dimensiones del contexto sociocultural y los medios digitales, para precisar el ámbito, las fortalezas y las debilidades epistemológicas y 
metodológicas del pensamiento computacional. En estas conclusiones precisamos los elementos de la complejidad educativa a la que el pensamiento computacional tiene que adecuarse y se pone en claro el entramado de problemas que ha evidenciado la literatura, la práctica educativa y el diseño de software.

En primer término, la evolución social, científica y cultural, y la crisis de algunos de los principios del neopositivismo nos han mostrado los límites del tecnocentrismo y del solucionismo tecnológico. Paradójicamente, estas limitaciones se presentan en vista de la sociedad red y de innovaciones como la inteligencia artificial y la robótica. Al respecto, el pensamiento computacional debe ayudar a los futuros profesionales y ciudadanos a moverse en un escenario impredecible y de múltiples choques sociales, culturales y ambientales. Los medios digitales son una causa de estas transformaciones, pero los saberes y las habilidades que se necesitarán exceden el dominio de las ciencias de la computación. Por esto, las humanidades son herramientas fundamentales.

En lo referido al contexto tecnológico, hemos analizado el proceso computacional, la complejidad del medio digital $\mathrm{y}$, al final, el software como medio digital propiamente dicho, identificando su naturaleza -en esencia creativa- de metamedio. El análisis conduce a determinar que el objetivo fundamental de una estrategia de desarrollo digital tecnológico inclusivo y sostenible es la producción de tecnología original. Hemos resaltado, a través del concepto de computational creativity, el rol primordial de las identidades y tradiciones culturales (la etnomatemática y la etnocomputación), en tanto conservan algo esencial para la creatividad: la disponibilidad de ingredientes únicos y diferentes. Para el pensamiento computacional, todo esto apela a contenidos más complejos que las ciencias de la computación y el modelo STEM. Incluso el paradigma STEAM parece insuficiente (Henriksen, 2017), pues la convergencia con el arte no se debería dar en sentido romántico, sino coherentemente con las propiedades de las ciencias de la complejidad y del software.

Estas conclusiones han servido para actualizar el modelo de pensamiento computacional vigente. Para comenzar, los contenidos del pensamiento computacional son efectivamente muy amplios y genéricos, pero hay poco que hacer. Por un lado, la complejidad del paisaje cultural tecnológico creado por los medios digitales es real. En este sentido, hemos propuesto dividir el campo del pensamiento computacional entre contenidos gené- 
ricos y de corte humanístico; es decir, la information literacy y los contenidos relativos a las ciencias de la computación, relacionados con la computational creativity. En el primer caso, el pensamiento computacional sería un contenido añadido a cada asignatura, con la tarea de traducir sus contenidos y procesos cognitivos propios para ser ejecutados por una máquina. En el segundo, hablamos de una asignatura más en las mallas curriculares. Además, es claro que las humanidades deben modernizar sus criterios, adaptándose a las características de los medios digitales, lo que todavía no sucede.

Por otro lado, esta complejidad nos pide también buscar soluciones tecnológicas y prácticas didácticas alternativas. Hemos propuesto recuperar los saberes y las habilidades tradicionales y analógicas, como el dibujo procedural o el algorítmico a mano alzada (figuras 5 y 6), y hemos presentado dos posibles soluciones, la máquina de Turing y las shape grammars, porque realizan el ideal de la cultura digital de combinar ciencia, arte, creatividad, naturaleza y tradiciones culturales (figuras 7 y 8).
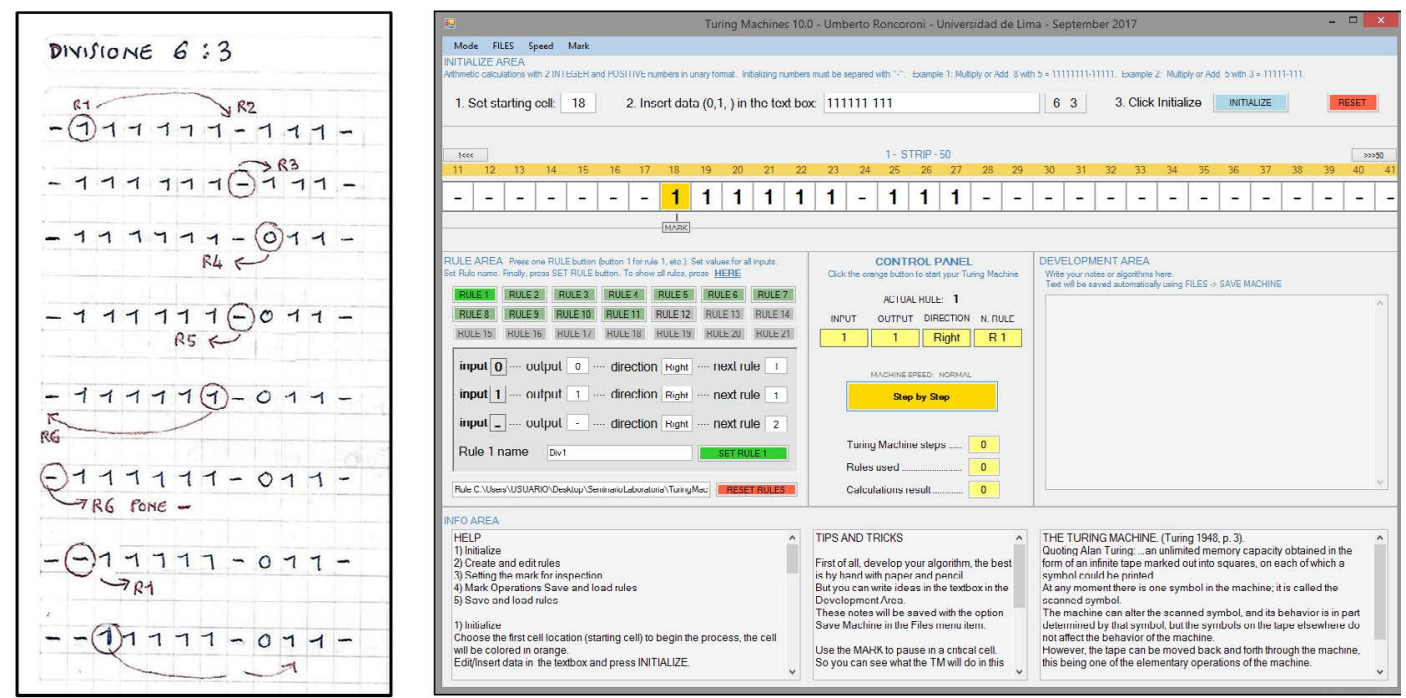

Figura 7: Izquierda: Desarrollo a mano de una máquina de Turing. Fuente: elaboración propia. A la derecha, Roncoroni, U. (2018). Turing machines 10.0. Software para el estudio y diseño de máquinas de Turing, desarrollado para el proyecto de investigación de la Universidad de Lima entre el 2018 y el 2019. La aplicación se puede descargar gratuitamente. Fuente: Digital Poiesis. 
INNOVACIÓN TEÓRICA

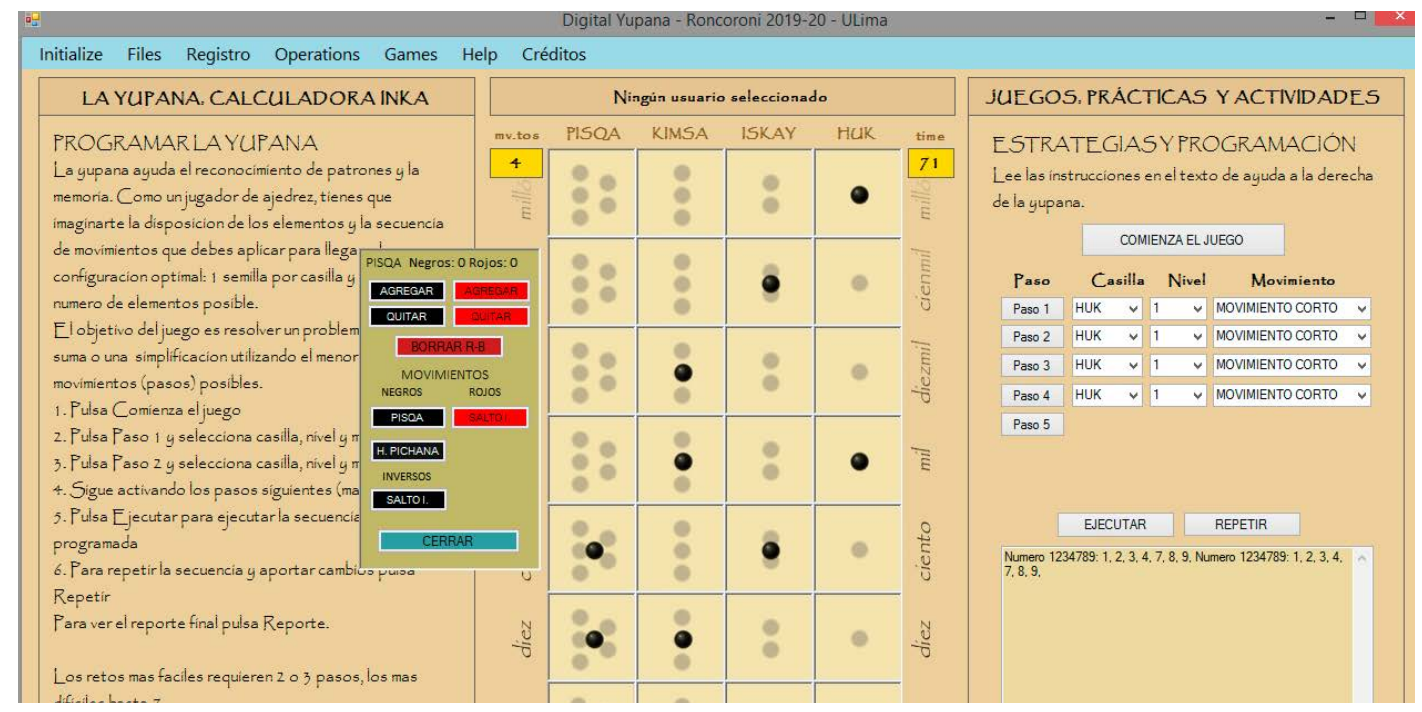

Figura 8: Roncoroni, U. (2019). Yupana digital. Software desarrollado en el marco del proyecto de investigación para la Universidad de Lima entre el 2018 y el 2019.

La aplicación se puede descargar gratuitamente. Fuente: Digital Poiesis.

Las ideas aquí planteadas no son las únicas posibles ni quizá las mejores. En todo caso, es posible concluir reafirmando un par de principios fundamentales y una recomendación para las instituciones educativas:

a. La computación y lo digital son hijos de ciertos principios, ideales y fines, de la historia y del contexto político y social. Estos contenidos son embebidos en los medios digitales que, por lo tanto, no deben ser utilizados como medios didácticos, pues sería como poner el carro antes que los bueyes.

b. El mayor poder educativo se obtiene por medio de las humanidades y los procesos naturales, desde el dibujo a mano alzada hasta el uso de materiales y artefactos analógicos. Es decir, para comprender los medios digitales y fortalecer el pensamiento computacional, ¡debemos limitar el uso de la tecnología!

Finalmente, los medios digitales son un reto mucho más complejo de lo que la literatura científica y las reformas educativas suponen; asimismo, es indispensable más investigación y experimentación para abordarlos de manera más consistente. 
No será una tarea fácil: si bien la retórica digital apela a la libertad de acceso y a la colaboración, en la práctica los medios digitales están en manos de monopolios que explotan tanto la tecnología como a los usuarios. Mejorar realmente la cultura tecnológica del ciudadano no está en el blanco del poder y del mercado, pues libera a los usuarios de la red a la que están vinculados. Nosotros, los educadores, debemos brindar a los estudiantes los aparatos críticos para moverse en este contexto y quizá sea este el compromiso más elevado del pensamiento computacional.

\section{Referencias}

Aho, A. V. (2012). Computation and computational thinking. Computer Journal, 55(7), 832-835.

Apaza Luque, J. (2017). La yupana, material educativo para la educación matemática. Madrid: Universidad Autónoma de Madrid.

Buckingham, D. (2006). Media education in the age of digital technology. 10th Anniversary MED Congress "La sapienza di comunicare". Recuperado de https:// www.academia.edu/2748203/.

Cobo Romaní, C., y Moravec, J. W. (2011). Aprendizaje invisible. Hacia una nueva ecología de la educación. Barcelona: Edicions de la Universitat de Barcelona.

Damasio, A. (2005). Descartes' Error. Nueva York: Penguin Books.

Deleuze, G. (2006). Postscriptum sobre las sociedades del control. Polis. Revista

Latinoamericana, 13. Recuperado de https://joumals.openedition.org/polis/5509.

Denning, P. J. (2017). Remaining trouble spots with computational thinking.

Communications of the ACM, 60(6), 33-39. doi: 10.1145/2998438.

Dussel, I., y Quevedo, A. (2010). Educación y nuevas tecnologías: los desafíos pedagógicos ante el mundo digital. En VI Foro Latinoamericano de Educación. Buenos Aires: Santillana.

Flake, G. W. (2000). The computational beauty of nature. Cambridge (Ma): The MIT Press. Flusser, V. (1984). Towards a philosophy of photography. Göttingen: European Photography. Fishwick, P. (Ed). (2008). Aesthetic computing. Cambridge (Ma): The MIT Press.

Fonseca, C. (2005). Educación, tecnologías digitales y poblaciones vulnerables: una aproximación a la realidad de América Latina y el Caribe. Consulta Regional del Programa Pan Américas IDRC. Recuperado de https://idl-bnc-idrc.dspacedirect. org/handle/10625/55410. 
Galanter, P. (2012). What is generative art? Complexity theory as a context for art. GA2003 - 6th Generative Art Conference proceedings. Recuperado de https:// www.generativeart.com/GA2012/phil.pdf.

Gil, A. (s. f.). Tipón, Perú. [Fotografía]. Recuperado de http://www.fotoaleph.com/ imagen-galeria.php/referencia/84110156/exposicion.

Guzdial, M., Kay, A., Norris, C., y Soloway, E. (2019). Computational thinking should just be good thinking. Communications of the ACM, 62(11), 28-30. doi: $10.1145 / 3363181$.

Han, B. C. (2014). En el enjambre. Buenos Aires: Herder.

Harris, K., Kimson A., y Schwede A. (2018). Labor 2030: The collision of demographics, automation, and inequality. Recuperado de http://www. bain.com/publications/articles/labor-2030-the-collision-of-demographicsautomation-and-inequality.aspx.

Henriksen, D. (2017). Creating STEAM with design thinking: Beyond STEM and arts integration. The STEAM Journal, 1(3). doi: 10.5642/steam.20170301.11

Johnson, L., Adams Becker, S., Estrada, V., y Freeman, A. (2015). NMC horizon report: 2015 higher education edition. Austin (TX): The New Media Consortium. Kahneman, D. (2012). Pensar rápido, pensar despacio. Barcelona: Debate.

Kay, A. (1984). Computer Software. Scientific American, 251(3), 52-59.

Kurz, R. (2003). La ignorancia de la sociedad del conocimiento. Recuperado de http://www.antroposmoderno.com/antro-articulo.php?id articulo=247.

Lanier, J. (2014). ¿Quién toma el control? Barcelona: Debate.

Lorenzo, E. (2019). Yupana. [Fotografía]. Recuperado de http://elieducacion. blogspot.com/2019/03/yupana-y-quipu.html.

Manovich, L. (2013). El software toma el mando. Barcelona: Fondo Editorial de la Universitat Oberta de Catalunya.

Mazzone, M., y Elgammal, A. (2019). Art, creativity, and the potential of artificial intelligence. Arts, 8(26). doi:10.3390/arts8010026.

Ministero dell' Istruzione, dell'Universitá e della Ricerca. (2010). La riforma della scuola secondaria superiore. Recuperado de http://archivio.pubblica. istruzione.it/riforma superiori/nuovesuperiori/index.html.

Morozov, E. (2014). La locura del solucionismo tecnológico. Buenos Aires: Katz.

Papert, S. (1980): Mindstorms: Children, computers, and powerful ideas. Nueva York: Basic Books. 
Prusinkiewicz, P., Cieslak, M., Ferraro, P., Hanan, J. (2018). Modeling plant development with L-systems. R. Morris (ed.), Mathematical modelling in plant biology (139-169). Recuperado de https://link.springer.com/chapter/10.1007/978-3-319-99070-5 8 .

Prusinkiewicz, P., et Al. (2018). Modeling plant development with L-systems. [Gráfico por computadora]. Recuperado de https://bit.ly/2WZPtdg

Rahimi, A. (2019). Machine learning is alchemy. Recuperado de https://yahnd. com/theater/r/youtube/x7psGHgatGM/.

Reimann, D., y Maday, C. (2017). Enseñanza y aprendizaje del modelado computacional en procesos creativos y contextos estéticos. Education in the knowledge society, 18(3), 87-97.

Rifkin, J. (2013). La era del acceso. La revolución de la nueva economía. Barcelona: Paidós.

Roncoroni, U. (2017-2019). Digital Poiesis. [Página web]. Recuperado de http:// www.digitalpoiesis.org.

Sansoni, G. (1930). Aeropittura. [Pintura.] Recuperado de https://www.italianways. $\mathrm{com} /$ tatos-dynamic-aerial-paintings/.

Schmidt-Crawford, D. (2018). Coding for teacher education: A recurring theme that requires our attention. Journal of Digital Learning in Teacher Education, 34(4). Recuperado de https://www.tandfonline.com/doi/full/10.1080/21532974.20 18.1499992.

Shapiro, J., y S. K. Hughes. (1996). Information literacy as a liberal art. Enlightenment proposal for a new curriculum. Educom Review, 31(2). Recuperado de https:// www.educause.edu/ir/library/html/erm/31231.html.

Shen, G. (2014). Shape grammar exercises. [Gráfico por computadora]. Recuperado de https://pt.slideshare.net/GiapSheng/shape-grammar-exercises.

Stallmann, R. (2002). Free software, free society. Boston: GNU Press.

Stiny, G, y J. Gips. (1971). Shape grammars and the generative specification of painting and sculpture. IFIP Congress, (71), 1460-1465. Recuperado de http:// www.cs.bc.edu/ gips/ShapeGrammarsIFIPS71.pdf.

Varma, R. (2006). Making computer science minority friendly. Communications of the ACM, 49(2) 129-134.

Wing, J. (2006). Computational thinking. Viewpoint. Communication of ACM, 49(3), 33-35. Recuperado de http://www.cs.cmu.edu/afs/cs/usr/wing/www/ publications/Wing06.pdf. 
Wolf, M. (2018). Reader, come home. The reading brain in a digital world. Nueva York: Harper \& Collins.

Zador, A. M. (2019). A critique of pure learning and what artificial neural networks can learn from animal brains. Nat Commun, 10(3770). Doi: 10.1038/s41467019-11786-6

\section{Notas}

[1] Se entiende por tecnocentrismo la postura que considera la educación tecnológica como simple capacitación en el uso de aparatos, y por solucionismo tecnológico, la creencia de que la tecnología, por sí misma, es una solución educativa eficaz y suficiente.

[2] La máquina de Turing, considerada el prototipo del proceso computacional, se basa en datos binarios y en reglas para su acceso, lectura y escritura. Las shape gramamars (Stiny y Gips, 1972) son procesos recursivos basados en la sustitución de símbolos alfanuméricos por secuencias de otros símbolos. Permiten generar formas complejas y fractales que se observan también en la naturaleza. Para más información, véase Digital Poiesis (http://www.digitalpoiesis.org).

[3] El taller experimental se dictó en la ONG educativa Laboratoria (https://www.laboratoria. la/), una startup ubicada en Lima, Perú, que se dedica a la formación tecnológica de mujeres de bajos recursos. Para el estudio de la máquina de Turing, de las etnomatemáticas y de las gramáticas generativas, véase Digital Poiesis, de donde es posible descargar las aplicaciones correspondientes.

[4] La yupana es la calculadora inca. Matemáticamente, es una máquina de estados finitos con principios similares a los de la máquina de Turing. Es una alternativa etnomatemática a esta última. Consiste en un tablero de cuatro columnas y $n$ filas (que corresponden a las unidades, decenas, centenares, etcétera) en el que los cálculos se hacen moviendo piezas (piedritas o semillas de maíz) de acuerdo a reglas, de modo similar a juegos como el ajedrez $o$ las damas chinas.

[5] "Generative art refers to any art practice where the artist uses a system, such as a set of natural language rules, a computer program, a machine, or other procedural invention, which is set into motion with some degree of autonomy contributing to or resulting in a completed work of art". (Galanter, 2003).

[6] No es una herramienta, a pesar de que actúa como diferentes herramientas. Es el primer metamedio y, como tal, tiene un nivel de libertad para la representación y la expresión que nunca antes ha sido encontrado y que todavía está poco estudiado. (Traducción de los autores).

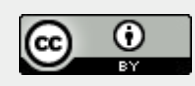

Este obra está bajo una licencia de Creative Commons Reconocimiento 4.0 Internacional. 